\title{
Human Over-the-Counter Drug Manufacturing
}

National Cancer Institute

\section{Source}

National Cancer Institute. Human Over-the-Counter Drug Manufacturing. NCI

Thesaurus. Code C106645.

The process of manufacturing non-prescription medications for humans by a pharmaceutical company. 